\title{
Transmuted Alpha Power Inverse Rayleigh Distribution: Properties and Application
}

\author{
A. S. Malik ${ }^{*}$, S. P. Ahmad \\ Department of Statistics, University of Kashmir, Srinagar, 190006, India \\ Received 14 November 2018, accepted in final revised form 14 February 2019
}

\begin{abstract}
This paper proposes a new three parameter-distribution through the technique known as Transmutation. The proposed distribution is named Transmuted Alpha power inverse Rayleigh Distribution. Several important properties of the distribution are derived. The parameter estimation is also carried out. Two real life data set are used at the end to describe the potential application of proposed model.
\end{abstract}

Keywords: Transmuted alpha power inverse Rayleigh distribution; Reliability; Entropy; Order statistics.

(C) 2019 JSR Publications. ISSN: 2070-0237 (Print); 2070-0245 (Online). All rights reserved. doi: http://dx.doi.org/10.3329/jsr.v11i2.38844

J. Sci. Res. 11 (2), 185-194 (2019)

\section{Introduction}

In recent years, there has been an increased interest among researchers to construct new flexible distribution by adding an additional parameter to the baseline distribution. An interesting technique to accomplish the task of parameter addition is transmutation. The Quadratic Rank Transmutation Map (QRTM) was suggested by Shaw and Buckley [1]. They used QRTM to construct non-Gaussian distribution. A number of authors have considered this generalization technique and successfully achieved efficiency over the base distribution. For example "Transmuted Lomax distribution" by Ashour and Eltehiwy [2], “Transmuted Weibull Distribution: A generalization of Weibull Distribution” by Aryal and Tsokos [3], "Transmuted power function distribution" by Haq et al. [4], "Transmuted New Weibull Pareto Distribution and its application" by Tahir et al. [5], "Transmuted Kumaraswamy Quasi Lindley Distribution with applications" by Elgarhy et al. [6], "Transmuted Pareto Distribution" by Merovci and Puka [7], "Transmuted Lindley Geometric distribution and its application" by Merovci and Elbatal [8], "Transmuted Weibull Power Function Distribution: its properties and applications " by Haq et al. [9], "A New Weibull Rayleigh Distribution with application to real life data" by Malik and

* Corresponding author: aaliyasayeed2@gmail.com 
Ahmad [10] etc. In this paper, Alpha Power Inverse Rayleigh Distribution (APIRD) suggested by Malik and Ahmad [11] is generalized using the QRTM. The proposed model is named as Transmuted Alpha Power Inverse Rayleigh Distribution (TAPIRD). Let $f(x)$ and $F(x)$ denote the pdf and cdf of base distribution, the cdf and pdf of generalized distribution are given by Eq. (1) and Eq. (2) respectively.

$$
\begin{aligned}
& G(x)=(1+\beta) F(x)-\beta(F(x))^{2} \quad ;|\beta| \leq 1 . \\
& g(x)=f(x)[1+\beta-2 \beta F(x)],
\end{aligned}
$$

where $\beta$ is the transmuted parameter.

The cdf and pdf of APIRD with scale parameters $\alpha>0$ and $\lambda>0$ are given by Eq. (3) and Eq. (4) respectively.

$$
\begin{aligned}
& F_{A P T}(x)= \begin{cases}\frac{\left(\alpha^{e^{-\frac{\lambda}{x^{2}}}}-1\right)}{(\alpha-1)} & ; \alpha \neq 1 \\
e^{-\frac{\lambda}{x^{2}}} & ; \alpha=1\end{cases} \\
& f_{A P T}(x)= \begin{cases}\frac{\log \alpha}{(\alpha-1)} \frac{2 \lambda}{x^{3}} e^{-\frac{\lambda}{x^{2}}} \alpha^{e^{-\frac{\lambda}{x^{2}}}} & ; \alpha \neq 1 \\
\frac{2 \lambda}{x^{2}} e^{-\frac{\lambda}{x^{2}}} & ; \alpha=1\end{cases}
\end{aligned}
$$

The main motivation for considering TAPIRD is to add an additional parameter to the base distribution i.e., APIRD so that the flexibility of base distribution can be enhanced. The proposed distribution exhibits more complex shapes of hazard rate function. Therefore, the proposed distributions could be used to model diverse nature of data sets. Also the proposed distribution outperforms some well-known models with respect to two real life data sets. The rest of paper is unfolded as following: in section 2 , the pdf and cdf of proposed distribution are introduced, section 3 deals with the reliability analysis of TAPIRD. The expression for mixture representation of the pdf, statistical properties and entropy estimation of TAPIRD are obtained in section 4, 5 and 6 respectively. The expressions for order statistics are derived in section 7 . In section 8 , the estimation of parameters is accomplished through the technique of maximum likelihood estimation. The simulation study and application of TAPIRD in real life is discussed in section 10 and 11 respectively. Finally some discussions and conclusions are presented at the end.

\section{TAPIRD}

On substituting Eqs. (3) (4) in Eqs. (1) and (2), we obtain the cdf and pdf of TAPIRD as given by Eqs. (5) and (6) respectively. 


$$
\begin{aligned}
& g(x)= \begin{cases}\frac{\log \alpha}{(\alpha-1)} \frac{2 \lambda}{x^{3}} e^{-\lambda / x^{2}} \alpha^{-\frac{\lambda}{x^{2}}}\left\{1+\beta+\frac{2 \beta}{\alpha-1}-\left(\frac{2 \beta}{\alpha-1} \alpha^{e^{-\frac{\lambda}{x^{2}}}}\right)\right\} ; \alpha \neq 1 \\
\frac{2 \lambda}{x^{3}} e^{-\lambda / x^{2}}\left(1+\beta-2 \beta e^{-\lambda / x^{2}}\right) & ; \alpha=1\end{cases} \\
& G(x)=\left\{1-\left\{\begin{array}{c}
\frac{\left[(\alpha+\beta)-\alpha\left(1-\beta \alpha^{e^{-\frac{\lambda}{x^{2}}-1}}\right)\right]\left(\alpha-\alpha^{e^{-\frac{\lambda}{x^{2}}}}\right)}{(\alpha-1)^{2}} \\
(1-\beta) e^{-\frac{\lambda}{x^{2}}}+2 \beta e^{-\frac{2 \lambda}{x^{2}}}
\end{array}\right\} ; \alpha \neq 1\right.
\end{aligned}
$$

For $\alpha=1$, the Eq. (5) reduces to Transmuted Rayleigh Distribution (TRD) given by [12]. So, for the rest of the paper we will consider only the case for which $\alpha \neq 1$.

The graph of pdf and cdf of TAPIRD for different parameter combination are given by Figs. 1.
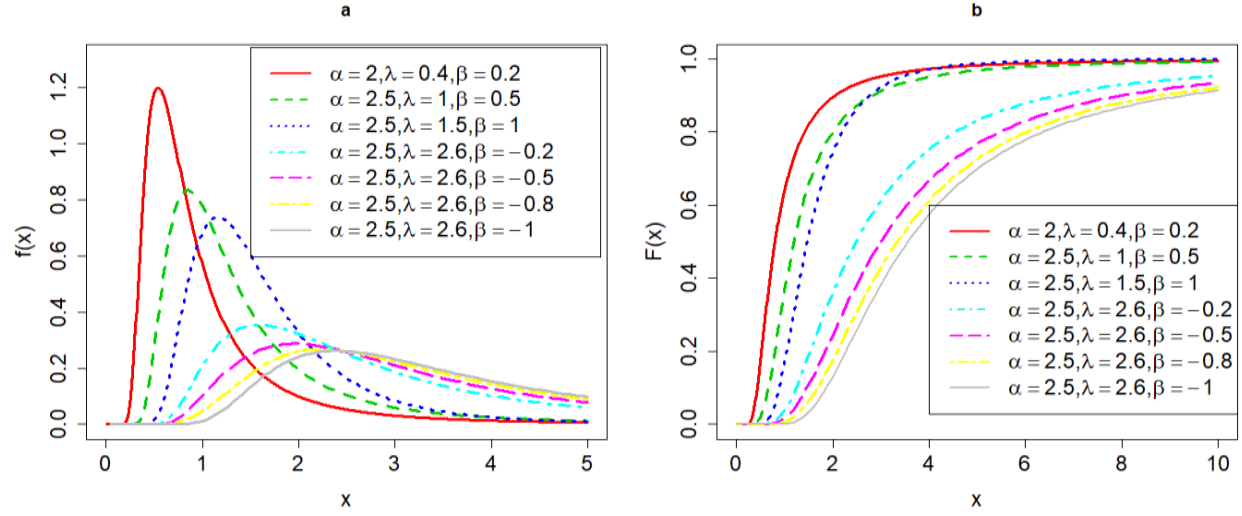

Fig. 1. Plots of (a) pdf and (b) cdf for TAPIRD with several parameter values.

Fig. 1 suggests that the proposed distribution is positively skewed and unimodal. The shape of pdf tends to flatten out for the negative values of the transmuted parameter and becomes peaked for positive values of the transmuted parameter. We further observe from Fig. 2 that the corresponding distribution function is an increasing function of $x$.

\section{Reliability Analysis}

\subsection{Reliability function}

The reliability function of TAPIRD is given as 


$$
R(x)=\left\{\frac{\left[(\alpha+\beta)-\alpha\left(1-\beta \alpha^{e^{-\frac{\lambda}{x^{2}}-1}}\right)\right]\left(\alpha-\alpha^{e^{-\frac{\lambda}{x^{2}}}}\right)}{(\alpha-1)^{2}}\right\}
$$

\subsection{Hazard function.}

The hazard rate function of TAPIRD is given as

$$
h(x)=\frac{2 \lambda \beta(\log \alpha) e^{-\frac{\lambda}{x^{2}}} \alpha^{-\frac{\lambda}{x^{2}}}}{x^{3}\left\{(\alpha-1)-\beta\left(\alpha^{e^{-\frac{\lambda}{x^{2}}}}-1\right)\right\}}-\frac{2 \lambda(\log \alpha) e^{-\frac{\lambda}{x^{2}}} \alpha^{e^{-\frac{\lambda}{x^{2}}}}}{x^{3} \alpha\left(1-\alpha^{e^{-\frac{\lambda}{x^{2}}}}\right)}
$$

\subsection{Mean residual life}

The mean residual life is defined as

$$
\mu(t)=\frac{1}{s(t)}\left[E(t)-\int_{0}^{t} x g(x) d x\right]-t
$$

Where $E(t)=\sum_{j=0}^{\infty} \frac{\Gamma\left(\frac{1}{2}\right)}{j !(j+1)^{\frac{1}{2}}} \frac{(\log \alpha)^{j+1}}{\alpha-1} \frac{2 \lambda^{\frac{1}{2}}}{t^{3}} e^{-(j+1) \lambda / x^{2}}\left\{1+\beta-\frac{2 \beta\left(2^{j}-1\right)}{\alpha-1}\right\}$.

On substituting Eq. (10) and Eq. (5) in Eq. (9) and simplifying, we get

$$
\mu(t)=\frac{1}{s(t)} \sum_{j=0}^{\infty} \frac{\lambda^{\frac{1}{2}}}{j !(j+1)^{\frac{1}{2}}} \frac{(\log \alpha)^{j+1}}{\alpha-1} e^{-(j+1) \lambda / x^{2}}\left\{1+\beta-\frac{2 \beta\left(2^{j}-1\right)}{\alpha-1}\right\} \gamma\left(\frac{1}{2}, \frac{(j+1) \lambda}{t^{2}}\right)-t .
$$

\subsection{Mean waiting time}

The mean waiting time is defined as

$$
\bar{\mu}(t)=t-\frac{1}{G(t)} \int_{0}^{t} x g(x) d x
$$

Upon substituting Eq. (5) and Eq. (6) and after simplification yields the below given expression

$$
\bar{\mu}(t)=t-\frac{1}{G(t)} \sum_{j=0}^{\infty} \frac{\lambda^{\frac{1}{2}} \Gamma\left(\frac{1}{2}, \frac{(j+1) \lambda}{t^{2}}\right)}{j !(j+1)^{\frac{1}{2}}} \frac{(\log \alpha)^{j+1}}{\alpha-1} e^{-(j+1) \lambda / x^{2}}\left\{1+\beta-\frac{2 \beta\left(2^{j}-1\right)}{\alpha-1}\right\} .
$$


The graph of hazard rate function for different parameter combinations is given by Fig. 2 .

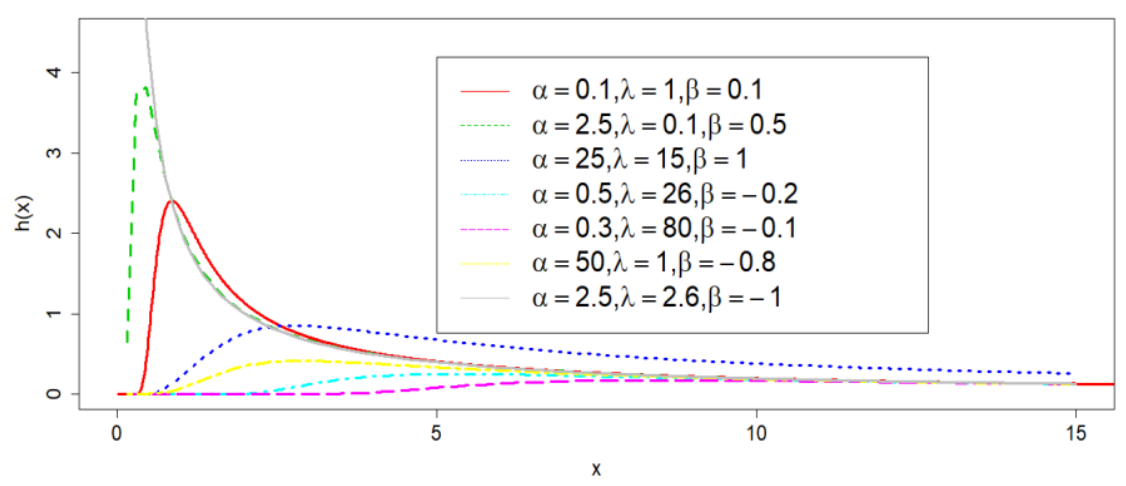

Fig. 2. Plot of hrf of TAPIRD.

Fig. 3 suggests that the proposed distribution is quite flexible in nature and can exhibit variety of shapes such as constant, reverse $\mathrm{j}$-shaped, upside-down bathtub shaped over the parameter space.

\section{Mixture Representation}

The pdf of TAPIRD can also be expressed as given below

$$
g(x)=\sum_{j=0}^{\infty} \frac{(\log \alpha)^{j+1}}{(\alpha-1) j !} \frac{2 \lambda}{x^{3}} e^{-(j+1) \lambda / x^{2}}\left\{1+\beta-\frac{2 \beta\left(2^{j}-1\right)}{\alpha-1}\right\} .
$$

The above given mixture representation is very useful in obtaining the properties of TAPIRD.

\section{Statistical Properties}

In this section, some statistical properties of TAPIRD are discussed.

\subsection{Moments}

The expression for $\mathrm{r}^{\text {th }}$ moment about origin of TAPIRD is given as

$$
\mu_{r}^{\prime}=\sum_{j=0}^{\infty} \frac{\Gamma\left(1-\frac{r}{2}\right)}{j !(j+1)}{ }^{1-\frac{r}{2}} \frac{(\log \alpha)^{j+1}}{\alpha-1} \frac{2 \lambda^{\frac{r}{2}}}{x^{3}} e^{-(j+1) \lambda / x^{2}}\left\{1+\beta-\frac{2 \beta\left(2^{j}-1\right)}{\alpha-1}\right\}: r<2
$$




\subsection{Incomplete moments about origin}

The expression for $\mathrm{s}^{\text {th }}$ incomplete moment about origin of TAPIRD is given as

$$
m_{s}(k)=\sum_{j=0}^{\infty} \frac{\Gamma\left(1-\frac{s}{2}, \frac{(j+1) \lambda}{k^{2}}\right)}{j !(j+1) \frac{s}{2}} \frac{(\log \alpha)^{j+1}}{\alpha-1} \frac{2 \lambda^{\frac{s}{2}}}{x^{3}} e^{-(j+1) \lambda / x^{2}}\left\{1+\beta-\frac{2 \beta\left(2^{j}-1\right)}{\alpha-1}\right\}: r<2 .
$$

Theorem 1. The moment generating function and characteristic function of a random variable $X$ following TAPIRD are respectively given by

$$
\begin{aligned}
& M_{X}(t)=\sum_{r=0}^{\infty} \sum_{j=0}^{\infty} \frac{t^{r} \Gamma\left(1-\frac{r}{2}\right)}{j ! r !(j+1)^{1-\frac{r}{2}}} \frac{(\log \alpha)^{j+1}}{\alpha-1} \frac{2 \lambda^{\frac{r}{2}}}{x^{3}} e^{-(j+1) \lambda / x^{2}}\left\{1+\beta-\frac{2 \beta\left(2^{j}-1\right)}{\alpha-1}\right\}: r<2 . \\
& \Psi_{X}(t)=\sum_{r=0}^{\infty} \sum_{j=0}^{\infty} \frac{(i t)^{r} \Gamma\left(1-\frac{r}{2}\right)}{j ! r !(j+1)^{1-\frac{r}{2}}} \frac{(\log \alpha)^{j+1}}{\alpha-1} \frac{2 \lambda^{\frac{r}{2}}}{x^{3}} e^{-(j+1) \lambda / x^{2}}\left\{1+\beta-\frac{2 \beta\left(2^{j}-1\right)}{\alpha-1}\right\}: r<2 .
\end{aligned}
$$

Proof. The moment generating function is given by

$$
\begin{aligned}
& M_{X}(t)=E\left[e^{t x}\right\rfloor \\
& M_{X}(t)=\int_{0}^{\infty} e^{t x} g(x) d x \\
& M_{X}(t)=\int_{0}^{\infty}\left(1+t x+\frac{(t x)^{2}}{2 !}+\cdots\right) g(x) d x \\
& M_{X}(t)=\sum_{l=0}^{\infty} \frac{t^{r}}{r !} \int_{0}^{\infty} x^{r} g(x) d x \\
& M_{X}(t)=\sum_{l=0}^{\infty} \frac{t^{r}}{r !} \mu_{r}^{\prime} \cdot \\
& M_{X}(t)=\sum_{r=0}^{\infty} \sum_{j=0}^{\infty} \frac{t^{r} \Gamma\left(1-\frac{r}{2}\right)}{j ! r !(j+1)} \frac{(\log \alpha)^{j+1}}{\alpha-1} \frac{2 \lambda^{\frac{r}{2}}}{x^{3}} e^{-(j+1) \lambda / x^{2}}\left\{1+\beta-\frac{2 \beta\left(2^{j}-1\right)}{\alpha-1}\right\}: r<2 .
\end{aligned}
$$

Also, we have

$$
\Psi_{X}(t)=M_{X}(i t)
$$

Therefore,

$$
\Psi_{X}(t)=\sum_{r=0}^{\infty} \sum_{j=0}^{\infty} \frac{(i t)^{r} \Gamma\left(1-\frac{r}{2}\right)}{j ! r !(j+1)^{1-\frac{r}{2}}} \frac{(\log \alpha)^{j+1}}{\alpha-1} \frac{2 \lambda^{\frac{r}{2}}}{x^{3}} e^{-(j+1) \lambda / x^{2}}\left\{1+\beta-\frac{2 \beta\left(2^{j}-1\right)}{\alpha-1}\right\}: r<2 .
$$




\section{Entropy}

The expression Renyi entropy of TAPIRD is given by Eq. (19)

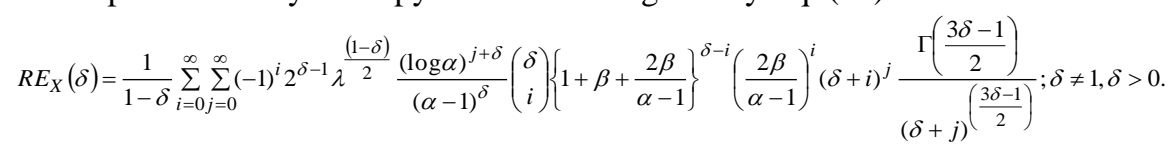

\section{Order Statistics}

Let $X_{1}, X_{2, \ldots} X_{n}$ be a random sample of size n drawn from TAPIRD and $X_{(1)}, X_{(2), \ldots} X_{(n)}$ be the corresponding order statistics. Then the pdf of $r^{\text {th }}$ order statistics denoted by $g_{\text {r: } n}(x)$ is given as

$$
\begin{aligned}
& \left.g_{r: n}(x)=\frac{n !}{(n-r) !(r-1) !}\left\{\left(\alpha+\beta-\alpha\left(1-\beta \alpha^{-\frac{\lambda}{x^{2}}}-1\right)\right)\left(\alpha-\alpha^{e^{-\frac{\lambda}{x^{2}}}}\right)\right\}^{n-r}\left\{(\alpha-1)^{2}-\left(\alpha+\beta-\alpha\left(1-\beta \alpha^{e^{-\frac{\lambda}{x^{2}}}-1}\right)\right)\right\}^{r-1}\right\} \\
& \left.\quad \frac{\log \alpha}{(\alpha-1)^{2 n}} \frac{2 \lambda}{x^{3}} e^{-\lambda / x^{2}} \alpha^{-\frac{\lambda}{x^{2}}}\right) \\
& -\frac{\lambda}{x^{2}}\left\{1+\beta+\frac{2 \beta}{\alpha-1}-\left(\frac{2 \beta}{\alpha-1} \alpha^{-\frac{\lambda}{x^{2}}}\right)\right\}
\end{aligned}
$$

Substituting $r=1$ and $r=n$ in Eq. (20) we get the pdf of first and $n^{\text {th }}$ order statistics as given by Eqn. (21) and Eqn. (22) respectively.

$$
\begin{aligned}
g_{1: n}(x)= & \left\{\left(\alpha+\beta-\alpha\left(1-\beta \alpha^{e^{-\frac{\lambda}{x^{2}}}-1}\right)\right)\left(\alpha-\alpha^{e^{-\frac{\lambda}{x^{2}}}}\right)\right\}^{n-1} \frac{\log \alpha}{(\alpha-1)^{2 n}} \frac{2 \lambda}{x^{3}} e^{-\lambda / x^{2}} \alpha^{e^{-\frac{\lambda}{x^{2}}}} \\
& \left\{1+\beta+\frac{2 \beta}{\alpha-1}-\left(\frac{2 \beta}{\alpha-1} \alpha^{-\frac{\lambda}{x^{2}}}\right)\right\} \\
g_{n: n}(x)= & n\left\{(\alpha-1)^{2}-\left(\alpha+\beta-\alpha\left(1-\beta \alpha^{e^{-\frac{\lambda}{x^{2}}}-1}\right)\right)\left(\alpha-\alpha^{e^{-\frac{\lambda}{x^{2}}}}\right)\right\} \frac{\log \alpha}{(\alpha-1)^{2 n}} \frac{2 \lambda}{x^{3}} e^{-\lambda / x^{2}} \alpha^{e^{-\frac{\lambda}{x^{2}}}} \\
& \left\{1+\beta+\frac{2 \beta}{\alpha-1}-\left(\frac{2 \beta}{\alpha-1} \alpha^{e^{-\frac{\lambda}{x^{2}}}}\right)\right\}
\end{aligned}
$$

\section{Parameter Estimation}

Let $X_{1}, X_{2, \ldots} X_{n}$ be a random sample of size $\mathrm{n}$ drawn from TAPIRD. Then, the $\log$ likelihood function is given by Eq. (23)

$$
\begin{aligned}
\log l= & n \log (\log \alpha)-n \log (\alpha-1)+n \log (2 \lambda)-3 \sum_{i=1}^{n} \log x_{i}-\sum_{i=1}^{n} \lambda / x_{i}^{2}+\sum_{i=1}^{n} e^{-\lambda / x_{i}^{2}} \log \alpha \\
& +\log \left\{1+\beta+\frac{2 \beta}{\alpha-1}-\left(\frac{2 \beta}{\alpha-1} \alpha^{-\frac{\lambda}{x^{2}}}\right)\right\} .
\end{aligned}
$$


Differentiating Eq. (18) w.r.t. $\alpha, \lambda$ and $\beta$ and equating to zero we get the maximum likelihood estimates of the parameters.

\section{Simulation Study}

In this section, a simulation study has been performed through $\mathrm{R}$ software to access the flexibility of proposed model. The values of the parameters are chosen to be $\alpha=3, \lambda=3$, and $\beta=0.3$.The data sets of size $25,75,150$ and 500 are obtained by using the inverse cdf method and the summary of results is presented in the Table 1 below:

Table 1. Estimates and performance of the distributions for simulated data.

\begin{tabular}{|c|c|c|c|c|c|c|c|c|}
\hline \multirow{3}{*}{$\begin{array}{l}\text { Sample } \\
\text { size }\end{array}$} & \multirow{3}{*}{ Distribution } & \multicolumn{3}{|c|}{ Estimates } & \multirow{3}{*}{$-\log 1$} & \multirow{3}{*}{ AIC } & \multirow{3}{*}{ BIC } & \multirow{3}{*}{ AICC } \\
\hline & & & & & & & & \\
\hline & & $\alpha$ & $\lambda$ & $\beta$ & & & & \\
\hline \multirow{4}{*}{25} & TAPIRD & 1.303 & 4.295 & -0.043 & 50.780 & 102.561 & 103.218 & 102.91 \\
\hline & APIRD & 1.397 & 4.314 & - & 51.782 & 107.56 & 110.000 & 107.918 \\
\hline & RD & - & 4.680 & - & 51.81 & 105.636 & 106.855 & 105.810 \\
\hline & TRD & - & 4.425 & 0.114 & 51.79 & 107.588 & 110.026 & 107.762 \\
\hline \multirow{4}{*}{75} & TAPIRD & 15.01 & 2.720 & 0.622 & 93.845 & 183.69 & 191.427 & 184.04 \\
\hline & APIRD & 2.331 & 3.340 & - & 94.325 & 192.65 & 196.47 & 193.00 \\
\hline & RD & - & 0.5934 & - & 94.58 & 191.172 & 193.08 & 191.34 \\
\hline & TRD & - & 1.388 & 0.642 & 94.21 & 192.433 & 196.257 & 192.607 \\
\hline \multirow{4}{*}{150} & TAPIRD & 3.512 & 3.778 & 0.551 & 271.91 & 541.826 & 548.85 & 542.17 \\
\hline & APIRD & 0.792 & 4.315 & - & 272.38 & 548.76 & 554.78 & 549.11 \\
\hline & $\mathrm{RD}$ & - & 4.072 & - & 272.46 & 546.92 & 549.93 & 547.09 \\
\hline & TRD & - & 4.352 & -0.1354 & 272.36 & 548.72 & 554.74 & 548.89 \\
\hline \multirow{4}{*}{500} & TAPIRD & 0.884 & 3.968 & 0.0192 & 920.01 & 1837.37 & 1849.01 & 1837.72 \\
\hline & APIRD & 0.852 & 3.966 & - & 920.68 & 1845.37 & 1853.80 & 1845.72 \\
\hline & RD & - & 3.814 & - & 920.82 & 1843.65 & 1847.86 & 1843.82 \\
\hline & TRD & - & 3.951 & -0.0721 & 920.69 & 1845.39 & 1853.82 & 1845.57 \\
\hline
\end{tabular}

\section{Application}

In order to access the flexibility of TAPIRD, two well established models are compared with TAPIRD and criterion such as AIC, BIC and AICC are used to select best model among the compared models for the considered real life data sets. The models used for comparison are Alpha Power Inverse Rayleigh Distribution (APIRD), Rayleigh Distribution (RD) given by Rayleigh [13] and TRD.

Data set 1: The data set 1 represent the survival times (in days) of 72 guinea pigs infected with virulent tubercle bacilli, observed and reported by Bjerkedal [14].

Table 1 shows maximum likelihood estimates and different information measures for models and Fig. 3 a shows the plots of the estimated pdfs of APIRD and other competitive models for data set 1 . 
Table 2. Estimates and performance of the distributions for data set 1.

\begin{tabular}{|c|c|c|c|c|c|c|c|}
\hline \multirow[b]{2}{*}{ Distribution } & \multicolumn{3}{|c|}{ Estimates } & \multirow[b]{2}{*}{$-\log 1$} & \multirow[b]{2}{*}{ AIC } & \multirow[b]{2}{*}{ BIC } & \multirow[b]{2}{*}{ AICC } \\
\hline & $\alpha$ & $\lambda$ & $\beta$ & & & & \\
\hline TAPIRD & 170.4 & 0.244 & 1.00 & 41.04 & 88.088 & 94.51 & 88.441 \\
\hline APIRD & 3918 & 0.146 & - & 55.54 & 115.09 & 119.37 & 115.26 \\
\hline RD & - & 1.790 & - & 54.65 & 111.31 & 113.45 & 111.48 \\
\hline TRD & - & 0.895 & 1.00 & 54.65 & 113.31 & 117.59 & 113.48 \\
\hline
\end{tabular}

Data set 2: The second data set is on the strengths of $1.5 \mathrm{~cm}$ glass fibres. The data was originally obtained by workers at the UK National Physical Laboratory and it has been used by Smith and Naylor [15].

Table 2 shows maximum likelihood estimates and different information measures for models and Fig. $3 \mathrm{~b}$ shows the plots of the estimated pdfs of APIRD and other competitive models for data set 2 .

Table 3. Estimates and performance of the distributions for data set 2.

\begin{tabular}{|c|c|c|c|c|c|c|c|}
\hline \multirow[b]{2}{*}{ Distribution } & \multicolumn{3}{|c|}{ Estimates } & \multirow[b]{2}{*}{$-\log 1$} & \multirow[b]{2}{*}{ AIC } & \multirow[b]{2}{*}{ BIC } & \multirow[b]{2}{*}{ AICC } \\
\hline & $\alpha$ & $\lambda$ & $\mathrm{B}$ & & & & \\
\hline TAPIRD & 6247 & 0.0905 & 0.7877 & 104.79 & 215.59 & 222.42 & 215.94 \\
\hline APIRD & 4557 & 0.0191 & - & 109.14 & 222.28 & 226.83 & 222.45 \\
\hline RD & - & 0.4629 & - & 163.75 & 329.51 & 331.79 & 329.69 \\
\hline TRD & - & 0.9417 & 0.3524 & 140.26 & 284.53 & 289.09 & 284.71 \\
\hline
\end{tabular}

It can be clearly seen from Tables 1 and 2 that TAPIRD has least value of AIC, BIC and AICC for both the data sets. Hence TAPIRD proves to be best model for the given data sets than the compared models.

(a)

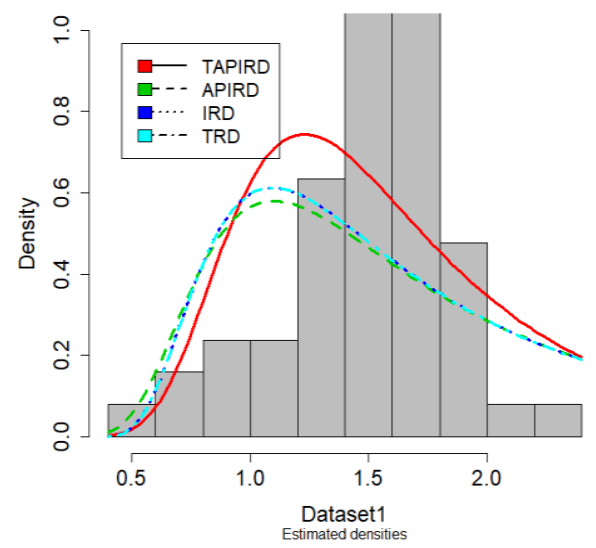

(b)

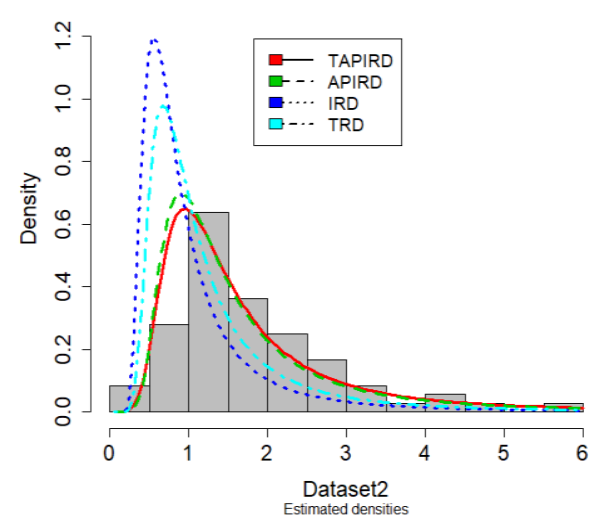

Fig. 3. (a) Plots of the estimated pdf of TAPIRD and other competitive models for data set 1 and (b) plots of the estimated pdf of TAPIRD and other competitive models for data set 2 . 


\section{Conclusion}

In this paper, a new model known as Transmuted Alpha Power Inverse Rayleigh Distribution has been successfully defined. The new distribution is more flexible as its hazard rate function exhibits more complex shapes. Two real life data sets were fitted for TAPIRD and compared with three known distributions. The results showed that the TAPIRD is a relatively better model to fit the data than the other three distributions. In simulation study, the same results were obtained. We hope that this distribution attracts wide variety of applications in diverse fields.

\section{References}

1. W. T. Shaw and L. R. C. Buckley, The Alchemy of Probability Distributions: Beyond GramCharlier Expansions, and a Skew-Kurtotic-Normal Distribution From a Rank Transmutation Map, Research report, (Financial Mathematics Group, King's College, London, UK, 2007), pp. $1-28$.

2. S. K. Ashour and M. A. Eltehiwy, Am. J. Appl. Math. Stat. 1, 6 (2013). https://doi.org/10.12691/ajams-1-6-3

3. G. A ryal and C. P. Tsokos, Eur. J. Pure Appl. Math. 4, 2 (2011).

4. M. A. Haq, N. S. Butt, R. M. Usman, and A.A. Fattah, Gazi University J. Sci. 29, 1 (2016).

5. A. Tahir, A. S. Akhter, A. S., M. A. Haq, Applications Appl. Math.-Int. J. 13, 1 (2018).

6. M. Elgarhy, I. Elbatal, M. A. U. Haq, and A. S. Hassan, Annals Data Sci. 5, 4 (2018).

7. F. Merovci and L. Puka, Prob Stat Forum 7, 1(2014).

8. F. Merovci and I. Elbatal, J. Stat. Appl. Prob. 3, 1 (2014). https://doi.org/10.18576/jsap/030107

9. M. A. Haq, M. Elgarhy, S. Hashmi, G. Ozel, and Q. U. Ain, J. Data Sci. 16, 2 (2018).

10. A. S. Malik and S. P. Ahmad, Int. J. Manag. Technol. Eng. 8, 12 (2018).

11. A. S. Malik and S. P. Ahmad, Int. J. Sci. Res. Math. Stat. 5, 5 (2018).

12. F. Merovci, Austrian J. Stat. 42, 1 (2013). https://doi.org/10.17713/ajs.v42i1.163

13. J. Rayleigh, Philos. Mag. 10, 73 (1980). https://doi.org/10.1080/14786448108627074

14. T. Bjerkedal, Am. J. Hyg. 72, 130 (1960).

15. R. L. Smith and J. C. Naylor, Appl. Stat. 36, 358 (1987). https://doi.org/10.2307/2347795 